\title{
Direct numerical simulation of three-dimensional inertial wave attractors
}

\author{
Ilias Sibgatullin*, Eugeny Ermanyuk ${ }^{\dagger}, \mathrm{Xu}$ Xiulin*, Leo Maas ${ }^{\ddagger}$, Thierry Dauxois ${ }^{\S}$ \\ * Moscow Lomonosov State University, Moscow, Russia \\ Email: sibgat@mech.math.msu.su, ilias.sibgat@gmail.com \\ * Ivannikov Institute for System Programming, Moscow, Russia \\ * Shirshov Institute of Oceanology, Moscow, Russia \\ $\dagger$ Lavrentyev Institute of Hydrodynamics, Novosibirsk, Russia \\ $\ddagger$ Institute for Marine and Atmospheric research Utrecht, Utrecht University, The Netherlands \\ $\S$ Univ Lyon, ENS de Lyon, Univ Claude Bernard CNRS, Laboratoire de Physique, Lyon, France
}

\begin{abstract}
Internal (inertial) waves in a uniformly stratified (rotating) fluid obey a highly specific dispersion relation that admits their propagation in form of oblique beams, which preserve their inclination to the distinguished direction (prescribed by gravity for internal waves and the angular velocity vector for the inertial waves) upon reflection. In confined domains with sloping walls, repeated reflections of the wave beams lead to concentration of the wave energy at closed loops called wave attractors. The dynamics of wave attractors is best studied in essentially twodimensional problems (plane or axisymmetric), progressing from the ideal-fluid concept to more realistic ones, with consideration of viscous effects, energy balance and cascades of wave-wave interactions. Development of fully three-dimensional highly nonlinear regimes has not yet been unexplored.
\end{abstract}

The present paper considers direct numerical simulations of inertial wave attractors in an axisymmetric rotating annulus having a trapezoidal cross section and a vertical axis of revolution. The rotating fluid volume is confined between two vertical coaxial cylinders, with truncated cone as a bottom surface. The large-scale forcing is applied to the fluid volume by specific motion of the upper lid. The spectral element method (based on Nek5000 open solver) is used to solve the Navier-Stokes equations in rotating fluid, with the non-slip boundary conditions at all rigid walls, and a prescribed vertical velocity field at the upper lid.

We consider two types of forcing. The first one simulates a small-amplitude nutation (Euler-disk-type motion) of the rigid lid, where the vector normal to the lid undergoes precession in such a way that the tip of the vector describes a horizontal circle of small radius around the axis of rotation of the annulus. This motion is modelled by prescribing the vertical velocity field with cosine-shaped running wave in azimuthal direction and linear variation in the radial direction. The response to such forcing mimics some essential features of tidal excitation. We show that attractors are formed only when the sense of nutation in azimuthal direction (in rotating coordinate system) is opposite to the sense of the background rotation (in a fixed laboratory system). In a horizontal cross-section of the flow we see then a rotating pattern with 'Yin-Yang' interplay in laminar mode, and when instability occurs with growth of the amplitude of external forcing, we see the interplay between the large- and small-scale 'Yin-Yang' patterns.

The second type of forcing is purely axisymmetric. At the upper lid we prescribe the vertical velocity profile in radial direction, with the amplitude in form of half-wave of the Bessel function, and simple harmonic time dependence. Such forcing excites a purely axisymmetric motion in linear regime. As the forcing increases, the axial symmetry of the inertial-wave motion

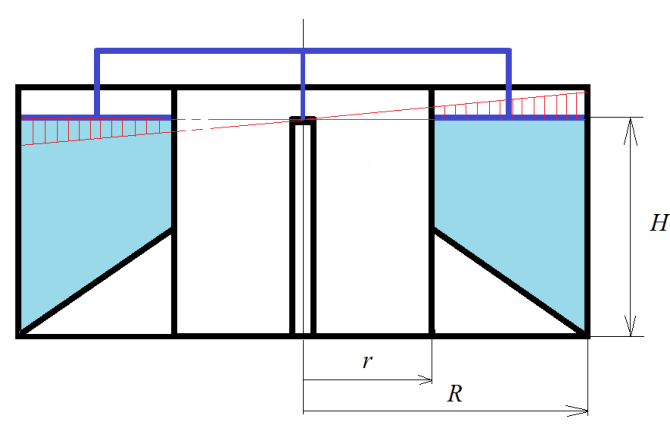

Figure 1. Scheme of the experimental setup

is broken: in the horizontal cross-section we observe the development of fine-scale 'Mandala' patterns possessing rotational symmetry whose complexity grow with time.

In both cases of forcing the triadic resonance is responsible for development of instability, and at sufficiently large forcing we observe a transition to three-dimensional wave turbulence. We show thus for the first time that fully three-dimensional simulations are necessary to capture the essential features of nonlinear regimes in inertial wave attractors in a rotating fluid annulus.

Index Terms-inertial waves, wave attractors, wave-wave interactions

\section{INTRODUCTION}

Wave motions in fluids, which are uniformly stratified in density and/or angular momentum, obey an anisotropic dispersion relation. This dispersion relation admits oblique wave beams as a generic form of wave motion. Being reflected at a rigid boundary, the wave beams preserve their angle of inclination with respect to the distinguished direction given by the gravity (angular velocity) vector for internal (inertial) waves, respectively. Reflection of wave beams at a sloping wall leads to strong decrease (focussing) or increase (defocussing) of their width. In closed fluid domains focussing prevails, leading to concentration of wave energy at wave attractors, whose linear "skeletons" can be found by tracing the wave-ray 


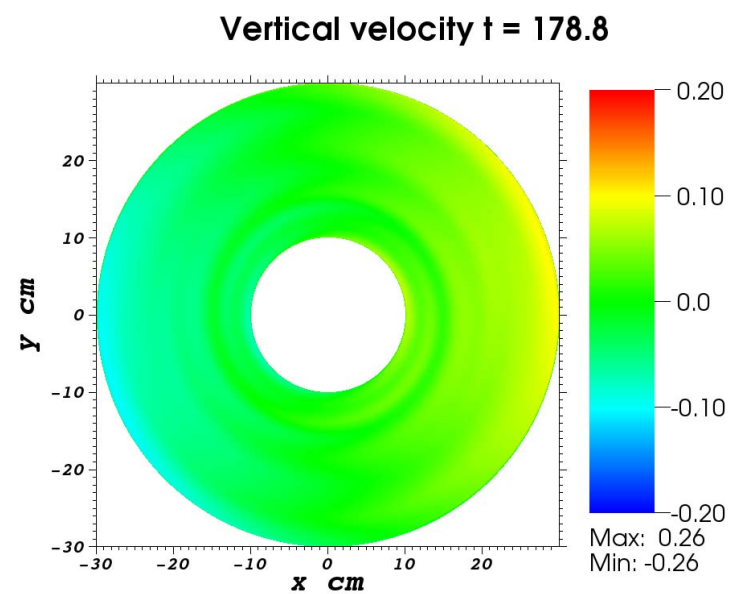

Figure 2. Vertical component of velocity in the slice $z=-15 \mathrm{~cm}$, amplitude $a=0.1 \mathrm{~cm}, \omega_{0}=2.4 \mathrm{rad} / \mathrm{s}, \Omega=2 \mathrm{rad} / \mathrm{s}$ for positive nutation.

Vertical velocity $t=178.8$

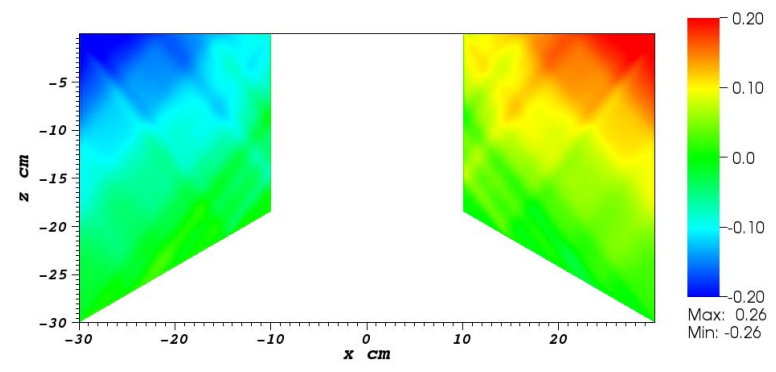

Figure 3. Vertical component of velocity in the slice $y=0$, amplitude $a=0.1 \mathrm{~cm}, \omega_{0}=2.4 \mathrm{rad} / \mathrm{s}, \Omega=2 \mathrm{rad} / \mathrm{s}$ for positive nutation.

billiard. The 2D internal wave attractors have been described in [24], [25]. Since then their relevance has been recognized in numerous problems of geophysical and astrophysical hydrodynamics, where an extensive literature describes attractors in rotating spherical liquid shells [16], [33], [38]. In ideal fluid, wave attractors represent singular structures, with infinite concentration of wave energy at their infinitely thin branches. The regularization of the problem requires the presence of a dissipative mechanism, which can be linear (purely viscous) [17], [18], [33]-[35], or more general [2], [32], where viscosity acts in combination with wave-wave interactions [8][10], [12], [36] and wall friction [4].

Numerical simulations of wave attractors are quite complicated. In numerical simulations of linear regimes one needs to resolve the boundary layers at rigid boundaries, and the structure of the attractor beams, which appear in form of oblique viscous shear layers. In simulations of strongly nonlinear regimes, one needs to resolve the small-scale patterns resulting from a cascade of wave-wave interactions. In a densitystratified fluid the numerical simulations are complicated by necessity to resolve diffusion of the stratifying agent.

It is important to note that the vast majority of studies

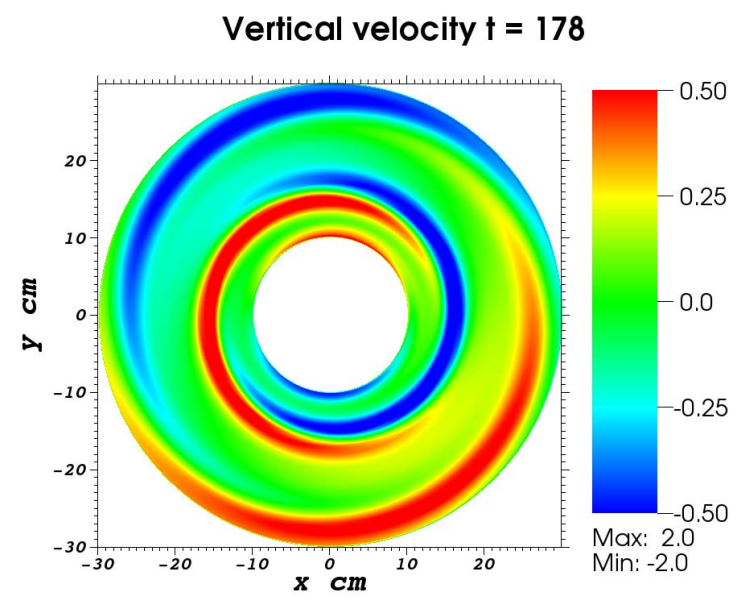

Figure 4. Vertical component of velocity in the slice $z=-15 \mathrm{~cm}$, amplitude $a=0.1 \mathrm{~cm}, \omega_{0}=2.4 \mathrm{rad} / \mathrm{s}, \Omega=2 \mathrm{rad} / \mathrm{s}$ for negative nutation.

Vertical velocity $\mathrm{t}=177.8$

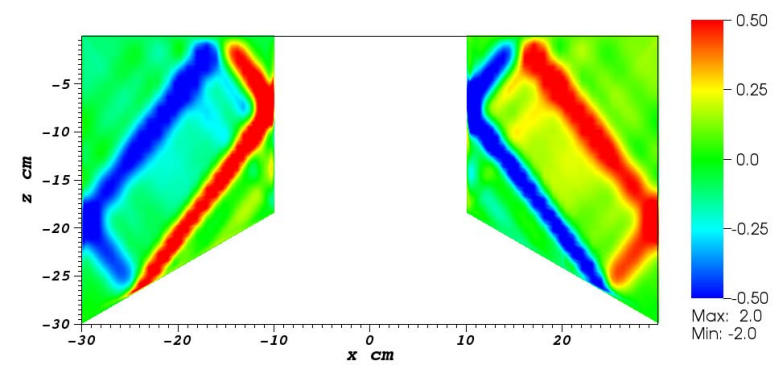

Figure 5. Vertical component of velocity in the slice $y=0$, amplitude $a=0.1 \mathrm{~cm}, \omega_{0}=2.4 \mathrm{rad} / \mathrm{s}, \Omega=2 \mathrm{rad} / \mathrm{s}$ for negative nutation.

on wave attractors is concerned with two-dimensional (plane or axisymmetric) problems. Existing studies of 3D problems consider geometrical configurations of wave attractors which are compatible with a specific shape of the fluid volume [14], [19], [27]. In the present paper we take another look at the three-dimensional effects. We consider an axisymmetric fluid volume, a rotating annulus with a trapezoidal crosssection as a generatrix. Previous studies of inertial waves in a rotating annulus, with perturbations introduced by angular oscillations of the inner cylinder, were focussed at linear and weakly nonlinear effects [21]. We apply a strong forcing to the upper boundary of the rotating annulus (physically this can be done with a version of the apparatus described in [29]) and explore via direct numerical simulations how the inertial-wave patterns possessing rotational or purely axial symmetry in linear regime evolve toward highly complex nonaxisymmetric small-scale wave patterns in nonlinear regime. The direct numerical simulations are performed with Nek5000 open solver implementing the spectral element method.

The paper is organized as follows. In section 2 we introduce the mathematical formulation of the problem and describe the geometric setup and the numerical procedure. In Section 


\section{Vertical velocity $t=141.8$}
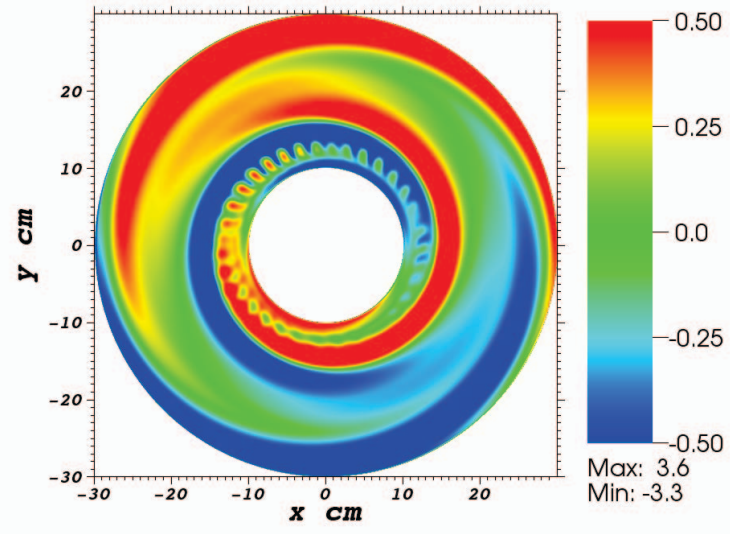

Figure 6. Vertical component of velocity in plane $z=-15 \mathrm{~cm}$, at amplitude $a=0.15 \mathrm{~cm}$

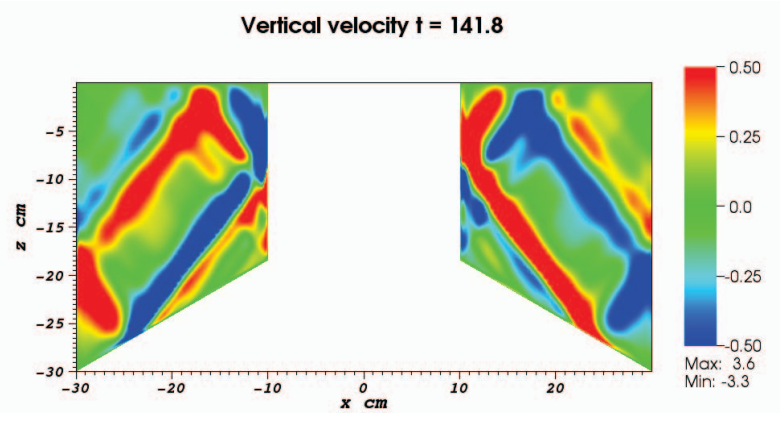

Figure 7. Vertical component of velocity in plane $y=0$, at amplitude $a=$ $0.15 \mathrm{~cm}$

3 we describe numerical results for two types of forcing in weakly and strongly nonlinear regimes, with special emphasis on purely three-dimensional features emerging in the wave pattern as result of triadic resonance. Brief conclusions are presented in Section 4.

\section{MAThematical formulation OF THE PROBlem}

Let us consider a rotating annulus filled with an incompressible fluid of density $\rho$. The annulus has a trapezoidal crosssection as a generatrix. Thus, the fluid volume is delimited by vertical walls represented by two coaxial vertical cylinders, and a bottom in form of a truncated cone. Such a geometry due to its periodic nature is able to avoid some complexities encountered in a nontrivial singly-connected fluid domain, as the rectangular trapezoids considered in [26]-[28]. The forcing at the upper boundary is applied by a lid executing a prescribed motion as discussed below in more detail. Without perturbations, the fluid in the annulus undergoes a uniform rigid-body rotation around the vertical axis of symmetry with constant angular velocity $\boldsymbol{\Omega}$. The scheme of the setup is shown in Fig. 1. Such a geometry is of importance for modelling dynamics of geophysical rotating layers. A reader interested in this subject is referred to recent papers on this subject [5]-[7],

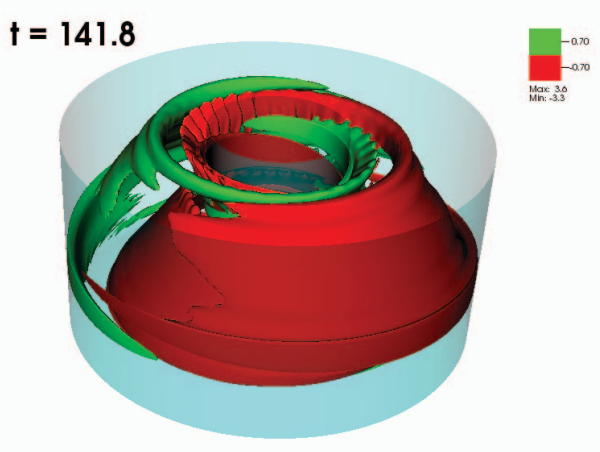

Figure 8. Two contours of the vertical component of velocity, appearance of the small scale daughter waves is clearly visible. Amplitude $a=0.15 \mathrm{~cm}$.

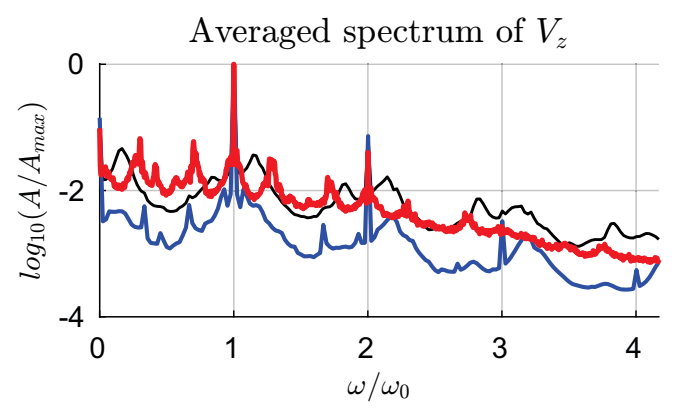

Figure 9. Evolution of the vertical velocity spectrum. The spectrum is averaged over the area of maximum amplitude near the first ray of the attractor. The blue line represents the initial stage of instability; next we have the transitional black line with one minimum below the external frequency $\omega_{0}$, and finally the red line corresponds to the fully developed regime. Amplitude of the external forcing is $a=0.15 \mathrm{~cm}$.

[11], [20], [23], as well as preceding ones [1], [3], [22], [37], which, however, do not consider highly nonlinear regimes that are of central interest in this study.

This work anticipates the experimental laboratory model in spirit of [29]. Therefore we will stick to dimensional variables with internal radius $R_{1}$ equal to $10 \mathrm{~cm}$, the outer radius $R_{2}$ equal to $30 \mathrm{~cm}$, and the maximum depth of the fluid volume $H$ is $30 \mathrm{~cm}$. The angle between the sloping generatrix of the conical bottom and the horizontal is set at $30^{\circ}$. The rate of background rigid-body rotation is $\Omega=2 \mathrm{rad} / \mathrm{s}$.

The mathematical model consist of Navier-Stokes equations, continuity equation and the boundary conditions imposed on the velocity field:

$$
\begin{gathered}
\frac{\partial \vec{v}}{\partial t}+v^{k} \nabla_{k} \vec{v}=-\nabla \tilde{p}+\nu \Delta \vec{v}+2 \boldsymbol{\Omega} \times \vec{v} \\
\tilde{p}=\frac{p}{\rho}-\frac{1}{2}|\boldsymbol{\Omega} \times \vec{r}|^{2}
\end{gathered}
$$




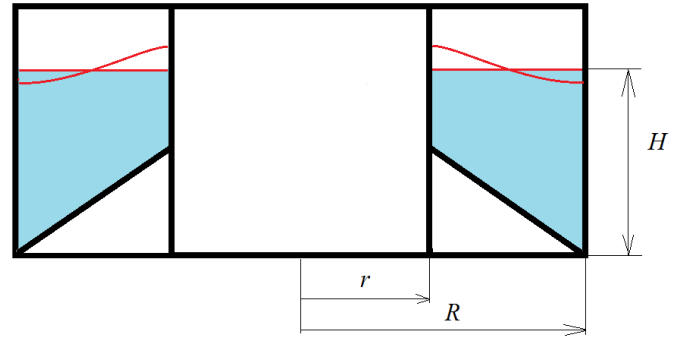

Figure 10. Scheme of an axisymmetric forcing

$$
\operatorname{div} \vec{v}=0
$$

We prescribe the no-slip condition at all rigid boundaries except the upper lid where we apply a specific harmonic forcing. Here we consider the following three cases of the time-dependent form of the upper surface:

(A) Positive nutation

$$
z=a\left(x \cos \left(\omega_{0} t\right)+y \sin \left(\omega_{0} t\right)\right) / R_{2}
$$

(B) Negative nutation

$$
z=a\left(x \cos \left(\omega_{0} t\right)-y \sin \left(\omega_{0} t\right)\right) / R_{2}
$$

(C) Axially symmetric perturbation, where the vertical amplitude as function of the radial coordinate is prescribed in form of half-wave of the Bessel function (see Fig. 10 with simple harmonic time-dependence at each radial location.

The above expressions are written in the Cartesian system $(x, y, z)$ co-rotating with the setup, where $z$-axis is pointing upwards and coincides with the axis of rotation. The origin of the coordinate system is taken at the center of the upper lid. The upper lid represents an Euler disk, which undergoes a small-amplitude nutation with respect to the horizontal position in such a way that the vector normal to the lid is inclined at angle $\arctan \left(a / R_{2}\right)$ to the vertical so that the tip of this vector describes a horizontal circle around the axis of rotation. It is important to note that the rigid lid has no inherent rotation in the rotating system $(x, y, z)$. In the fixed laboratory system of coordinates we define the anti-clockwise background rotation $\Omega$ as positive. We define nutation as positive (negative) if the tip of the vector normal to the lid undergoes anti-clockwise (clockwise) precession around $z$-axis in the rotating frame $(x, y, z)$. In numerical simulations the nutation of the upper lid as well as the Bessel-wave forcing are implemented by prescribing the appropriate vertical velocity field at the upper horizontal boundary. Since we consider the small-amplitude input perturbations, such approach is fully justified. In simulations of internal wave attractors with similar implementation of the input forcing, we observed excellent agreement between numerical and experimental results [10].

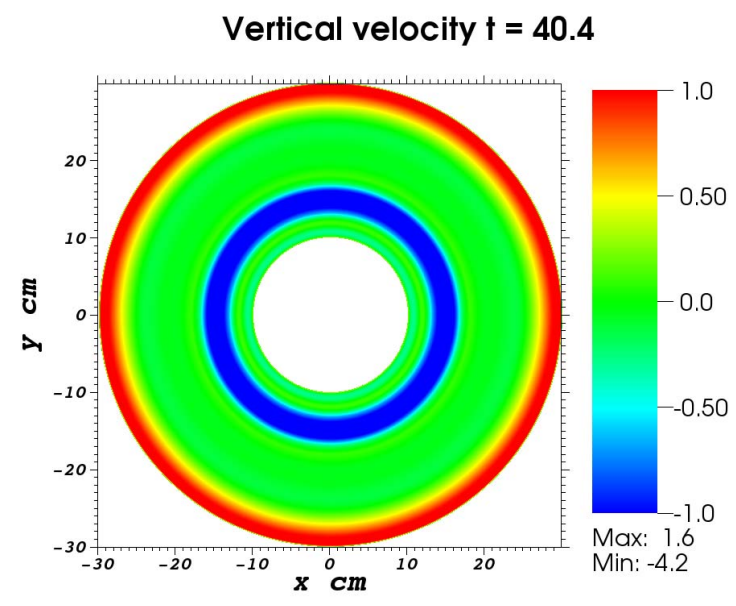

Figure 11. Vertical component of velocity in axisymmetric perturbation case in plane $z=-15 \mathrm{~cm}$

Vertical velocity $\uparrow=\mathbf{4 0 . 4}$

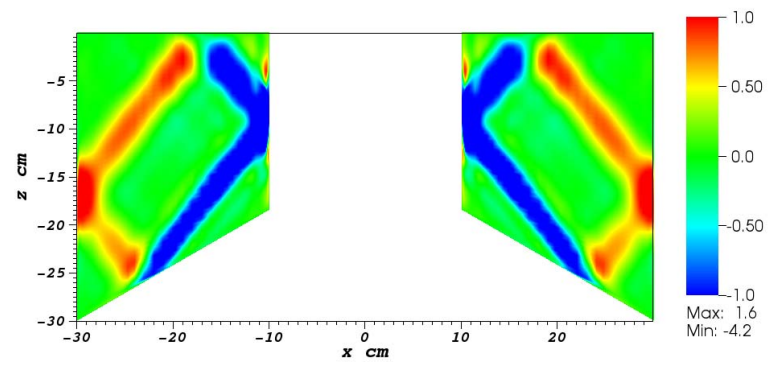

Figure 12. Vertical component of velocity in axisymmetric perturbation case in plane $y=0$

To model the development of instabilities and turbulence in strongly nonlinear regimes we need to take into account both viscous effects and nonlinear terms. Numerical simulation of transient and turbulent regimes is a challenge as we have to follow the development of small-scale structures at long time intervals. Spectral or Galerkin decomposition allows to account for nonlinear effects without parasitic effects due to numerical viscosity, but such a decomposition is possible only for a very limited number of geometrical settings and boundary conditions. In the present work we have performed the direct numerical simulations with the help of spectral element approach and open source code Nek5000 (see [13], [15]), which combines the advantages of high-order decomposition and geometric flexibility. The implementation of the method requires considerable efforts in comparison to conventional finite-volume approach and OpenFOAM. However, these efforts are fully justified by accuracy of long-term simulations of strongly nonlinear dynamics.

\section{NUMERICAL RESULTS AND INTERPRETATION}

\section{A. Forcing via "positive" nutation}

Figures 2, 3 show the snapshots of the field of the vertical component of velocity in horizontal cross-section $z=-15 \mathrm{~cm}$ 


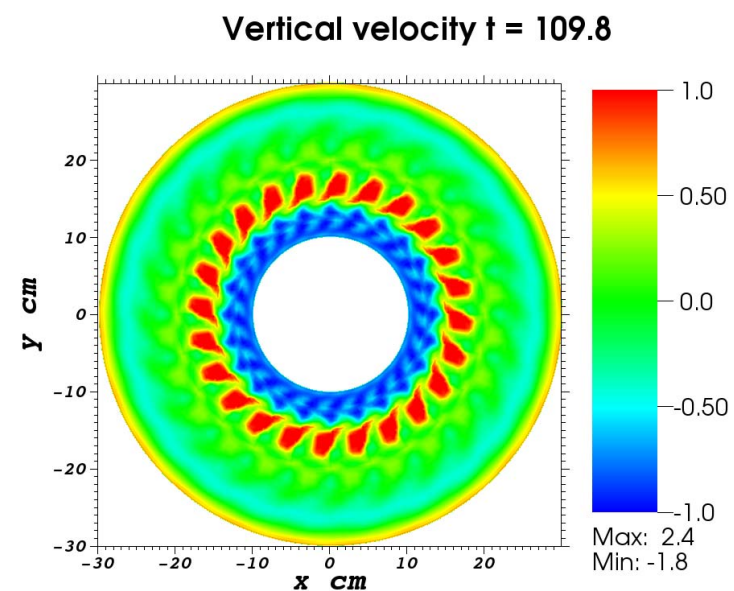

Figure 13. Snapshot of the vertical velocity field in horizontal slice $z=$ $-15 \mathrm{~cm}$.

Vertical velocity $t=109.8$

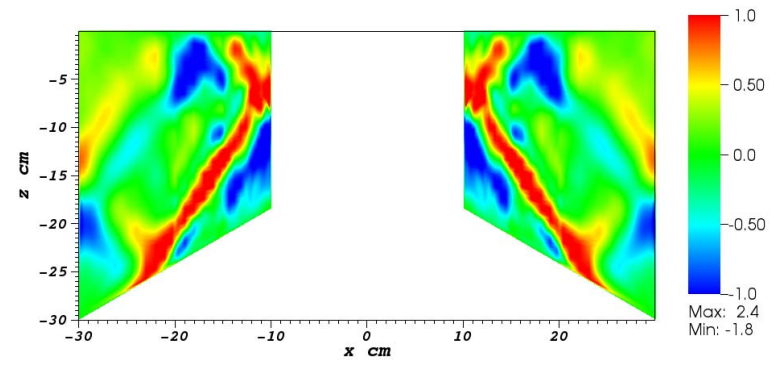

Figure 14. Snapshot of the vertical velocity field at vertical plane $y=0$.

and vertical cross-section $y=0 \mathrm{~cm}$. The snapshot is taken in the steady-state regime at $\omega_{0} t=2 \pi n$, where $n$ is a large integer number. The pattern is anti-symmetric with respect to plane $(y z)$ in agreement with the phase of the forcing imposed at the upper boundary. Some traces of inertial waves are detectable, but the overall pattern shows no signature of wave attractor. Note that the scale bar in these pictures is different from the scale bar used for the case of "negative" nutation depicted in figures 4 and 5 , in spite of the fact that in both cases the amplitude and frequency of nutation are the same. Thus, the case of forcing via "positive" nutation is not compatible with generation of inertial wave attractors.

\section{B. Forcing via "negative" nutation}

1) Linear regime (forcing amplitude $a=0.1 \mathrm{~cm}$ ): In contrast to 'positive nutation' considered in previous section, for 'negative nutation' we observe a completely different pattern of inertial wave motion. The snapshots of the vertical velocity fields presented in figures 4, 5 show well-developed wave attractors. Notice that the maximum amplitude of vertical velocity in this case is 8 times higher than in the case of 'positive nutation'! The wave attractor is in linear regime here. Obviously the wave attractor is not two-dimensional (axi-symmetric) since the left and right parts in Fig. 5 are

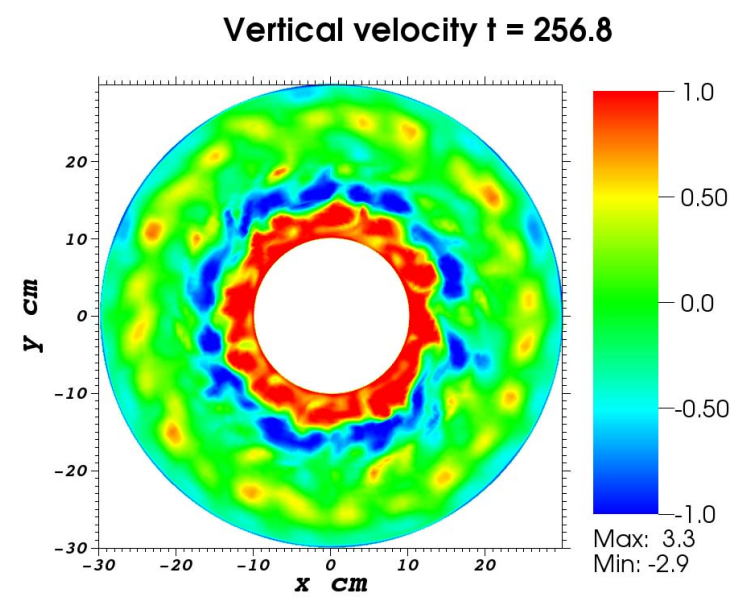

Figure 15. Snapshot of the vertical velocity field in horizontal slice $z=$ $-15 \mathrm{~cm}$.

Vertical velocity $t=256.8$

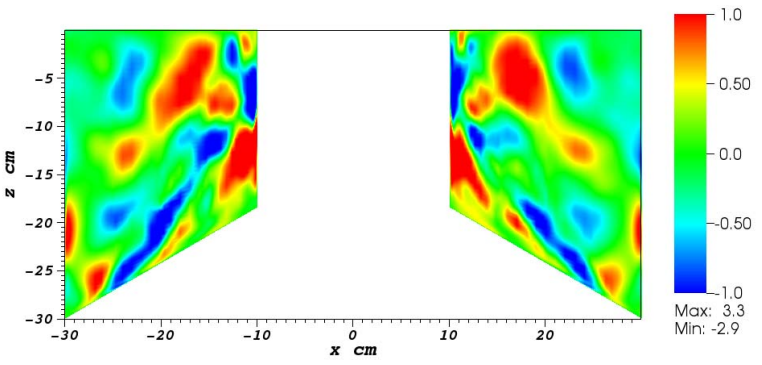

Figure 16. Snapshot of the vertical velocity field at vertical plane $y=0$.

in anti phase. In each horizontal cross-section (see e.g. Fig. 4) the wave motion appears as an 'Yin-Yang' pattern rotating in clockwise direction. In azimuthal direction the wavelength of the rotating pattern is equal to $2 \pi$, i.e. there is exactly one wave length per circumference in full agreement with the imposed forcing.

2) Nonlinear regime (forcing amplitude $a=0.15 \mathrm{~cm}$ ): As the forcing increases, we observe the appearance of smallscale secondary waves as shown in Fig. 6. It is worth noting an important difference in development of instability in the case of inertial waves in rotating annulus as compared to the 2D case of internal waves in a trapezoidal domain described in [36]. Indeed, in the 2D plane case the wave amplitude is the largest after the focusing reflection at the sloping boundary. It gradually decreases with distance from the focussing reflection due to viscosity. The instability develops in the most energetic branch of the wave attractor, creating favorable conditions for decomposition of the wave pattern into components with the help of Hilbert transform technique [30], [31], [36]. In inertial wave attractor the amplitude does not reach maximum after the focussing reflection at the sloping bottom. Instead, second focussing mechanism comes into play: the inertial wave converges toward the inner cylinder where it 
reaches the maximum amplitude. Viscosity acts in opposition to such amplification via convergence but the viscous decay is low under the conditions chosen for numerical experiments. Therefore, the instability occurs close to the surface of the inner cylinder, creating unfavorable conditions for the Hilbert decomposition in the vertical plane. Instead, we apply the Fourier and Hilbert analysis to the wave fields measured in horizontal cross-sections of the inertial wave fields.

First, we consider the frequency spectra. Figure 9 presents three spectra averaged over a ring-shaped zone at $z=-15 \mathrm{~cm}$ in the region corresponding to the maximum velocity amplitude, where the blue line corresponds to the onset of triadic resonance, the black line corresponds to transition to turbulence, and the red line corresponds to a developed regime of wave turbulence. The frequencies are normalized by the forcing frequency $\omega_{0}$. It can be seen that two secondary peaks are present in the subharmonical range of the first (blue) line at the spectrum corresponding to the onset of instability at $\omega_{1} / \omega_{0}=0.33$ and $\omega_{2} / \omega_{0}=0.67$. These peaks satisfy the classic temporal condition of triadic resonance $\omega_{1}+\omega_{2}=\omega_{0}$. With the Hilbert filtering performed in the horizontal plane we can determine the number of secondary waves per circumference travelling in clockwise and anticlockwise direction in the rotating reference frame. The filtered images of phase of the Hilbert transform show that the frequency peak at $\omega_{1}$ (resp. $\omega_{2}$ ) corresponds to secondary wave with azimuthal "wavelength" of $2 \pi / M$ (resp. $2 \pi / N$ ), where $M=38$ and $N=37$. Since for the primary wave we have the azimuthal "wavelength" of $2 \pi$, the spatial condition of triadic resonance translates into $M-N=1$. Thus, we observe an extremely efficient energy transfer from large to small scales. Note that the spectrum presented in figure 9 has a considerable discrete component at frequencies corresponding to multiples of the forcing frequency. In particular, the wave pattern filtered at frequency $2 \omega_{0}$ corresponds to the wave, which is twice shorter than the primary wave, with azimuthal "wavelength" equal to $\pi$. The three-dimensional structure of the unstable attractor at the onset of instability is presented in Fig. 8, where the secondary waves are clearly visible. As the instability develops, the frequency spectrum evolves in such a way that the relative importance of the discrete components decreases, while the continuous part of the spectrum increases (see figure 9). Finally, the 0-frequency peak implies driving of a mean flow, its spatial structure and comparison to [26] is the subject of current research.

\section{Axisymmetric forcing}

Let us now consider the axisymmetric forcing sketched in Fig. 10. Technically, such a forcing can be realized experimentally with a version of wave generator described in [29]. If we take the forcing amplitude $a=0.1 \mathrm{~cm}$, the fully symmetric attractor is first developed, but soon the instability sets in. Similar to the case described in the previous section, it is convenient to perform the Fourier and Hilbert analysis to the wave patterns observed in horizontal cross-sections. Similar to figure 9, figure 17 presents two spectra averaged over a

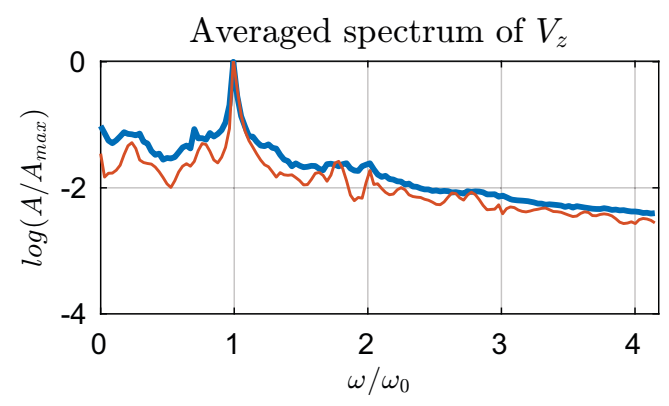

Figure 17. Spectrum of the vertical component of velocity in the point of maximum amplitude on the first ray of the attractor. Two peaks before the non dimensional external forcing frequency 1 are 0.23 and 0.77 . Red line corresponds to the initial stage of instability $t=10-60 \mathrm{~s}$. Blue line corresponds to the turbulent motion $t=210-300 \mathrm{~s}$.

ring-shaped zone at $z=-15 \mathrm{~cm}$. The spectra correspond, respectively, to the onset of triadic resonance and a more developed stage of instability. The frequencies are normalized by the forcing frequency $\omega_{0}$. Two secondary peaks are present in the subharmonical range at the spectrum (see red line) corresponding to the onset of instability at $\omega_{1} / \omega_{0}=0.23$ and $\omega_{2} / \omega_{0}=0.77$. Obviously, these peaks satisfy the temporal condition of triadic resonance. Applying the Hilbert filtering to the wave pattern measured in the horizontal plane, we observe that the frequency peak at $\omega_{1}$ (resp. $\omega_{2}$ ) corresponds to secondary wave with azimuthal "wavelength" of $2 \pi / M$ (resp. $2 \pi / N)$, where $M=N=22$. Since for the primary wave we have infinite azimuthal "wavelength", the spatial condition of triadic resonance translates into $M-N=0$. It is interesting that in the case of axisymmetric forcing the frequency spectrum is nearly continuous, especially in the case of welldeveloped instability. The development of instability in the horizontal cross-section creates first fascinating "Mandala"like patterns with the fine-scale elements possessing rotational symmetry of increasing complexity. As the instability develops toward wave turbulence, the fine-scale rotational symmetry is lost and the motion becomes chaotic. However, the presence of the wave attractor remains visible due to concentration of most energetic wave motions in the vicinity of its branches.

\section{CONCLUSION}

This paper presents a direct numerical simulation of inertial wave attractors in a rotating annulus with trapezoidal crosssection. Forcing is applied to the system by nutation of the lid which serves as the upper boundary of the annulus. We also consider a lid which undergoes a small purely axisymmetric deformation. This novel setup demonstrates a variety of linear and nonlinear regimes. We find that nutation-type forcing may or may not generate attractors depending on the sign of nutation with respect to the background rotation of the system. The attractor created by nutation has a three-dimensional structure, which appear in horizontal cross-sections as a rotating 'Yin- 
Yang' pattern. In nonlinear regime we observe the triadic resonance instability which effectively transfer energy from large to small scales. The pattern of secondary waves is fully threedimensional. In the case of axisymmetric forcing we observe an interesting scenario of instability which breaks the initially axisymmetric primary-wave pattern into small-scale fragments created by the interplay of secondary waves. These fragments possess a rotational symmetry of increasing complexity. At a later stage, the fine-scale rotational symmetry is lost, and we observe a transition to irregular three-dimensional wave pattern similar to wave turbulence.

\section{ACKNOWLEDGMENT}

This work is partially supported by RFBR grant 15-0106363, web-laboratory unihub.ru. The research is carried out using the equipment of the shared research facilities of HPC computing resources at Lomonosov Moscow State University. We would also thank A. Obabko, D. Ryazanov, K. Vatutin, V. Pryadun for useful discussions.

\section{REFERENCES}

[1] Keith D. Aldridge and Alar Toomre. Axisymmetric inertial oscillations of a fluid in a rotating spherical container. Journal of Fluid Mechanics, 37(2):307323, 1969.

[2] Auclair Desrotour, P., Mathis, S., and Le Poncin-Lafitte, C. Scaling laws to understand tidal dissipation in fluid planetary regions and stars i. rotation, stratification and thermal diffusivity. Astronomy and Astrophysics, 581:A118, 2015.

[3] R. C. Beardsley. An experimental study of inertial waves in a closed cone. Studies in Applied Mathematics, 49(2):187-196, 1970.

[4] F. Beckebanze, C. Brouzet, I. N. Sibgatullin, and L. R. M. Maas. Damping of quasi-2d internal wave attractors by rigid-wall friction. submitted to Journal of Fluid Mechanics, page arXiv:1707.08009, 2017.

[5] Ion Dan Borcia, Ghasemi V Abouzar, and U Harlander. Inertial wave mode excitation in a rotating annulus with partially librating boundaries. Fluid Dynamics Research, 46(4):041423, 2014.

[6] Ion Dan Borcia, Ghasemi V Abouzar, and U Harlander. Inertial wave mode excitation in a rotating annulus with partially librating boundaries. Fluid Dynamics Research, 46(4):041423, 2014.

[7] Ion Dan Borcia and Uwe Harlander. Inertial waves in a rotating annulus with inclined inner cylinder: comparing the spectrum of wave attractor frequency bands and the eigenspectrum in the limit of zero inclination. Theoretical and Computational Fluid Dynamics, 27(3):397-413, Jun 2013.

[8] C. Brouzet, I. N. Sibgatullin, E. V. Ermanyuk, S. Joubaud, and T. Dauxois. Scale effects in internal wave attractors. Phys. Rev. Fluids, 2:114803, Nov 2017.

[9] Christophe Brouzet, Evgeny Ermanyuk, Sylvain Joubaud, Ilias Sibgatullin, and Thierry Dauxois. Energy cascade in internal-wave attractors. EPL (Europhysics Letters), 113(4):44001, 2016.

[10] Christophe Brouzet, Ilias Sibgatullin, Helene Scolan, Evgeny Ermanyuk, and Thierry Dauxois. Internal wave attractors examined using laboratory experiments and 3d numerical simulations. Journal of Fluid Mechanics, 793:109-131, 2016.

[11] Zhen Cui, Keke Zhang, and Xinhao Liao. On the completeness of inertial wave modes in rotating annular channels. Geophysical \& Astrophysical Fluid Dynamics, 108(1):44-59, 2014.

[12] T. Dauxois, C. Brouzet, E. Ermanyuk, S. Joubaud, D. Le Tourneau, and I. Sibgatullin. Energy cascade in internal wave attractors. Procedia IUTAM, 20(Supplement C): 120 - 127, 2017. 24th International Congress of Theoretical and Applied Mechanics.

[13] M.O. Deville, P.F. Fischer, and E.H. Mund. High-Order Methods for Incompressible Fluid Flow. Cambridge Monographs on Applie. Cambridge University Press, 2002.

[14] Sybren Drijfhout and Leo Maas. Impact of channel geometry and rotation on the trapping of internal tides. Journal of physical oceanography, 37(11):2740-2763, 2007.
[15] Paul F Fischer. An overlapping schwarz method for spectral element solution of the incompressible navierstokes equations. Journal of Computational Physics, 133(1):84 - 101, 1997.

[16] T. Gerkema, J. T. F. Zimmerman, L. R. M. Maas, and H. van Haren. Geophysical and astrophysical fluid dynamics beyond the traditional approximation. Reviews of Geophysics, 46(2).

[17] N. Grisouard, C. Staquet, and I. Pairaud. Numerical simulation of a two-dimensional internal wave attractor. Journal of Fluid Mechanics, 614:1, October 2008

[18] J. Hazewinkel, P. van Breevoort, S. Dalziel, and L.R.M. Maas. Observations on the wavenumber spectrum and evolution of an internal wave attractor. Journal of Fluid Mechanics, 598:373-382, 2008.

[19] Jeroen Hazewinkel, Leo Maas, and Stuart B Dalziel. Tomographic reconstruction of internal wave patterns in a paraboloid. Experiments in fluids, 50(2):247-258, 2011

[20] M. Klein, A. Ghasemi, U. Harlander, and A. Will. Inertial Wave Excitation and Wave Attractors in an Annular Tank: DNS. In $E G U$ General Assembly Conference Abstracts, volume 16 of EGU General Assembly Conference Abstracts, page 15585, May 2014.

[21] Marten Klein, Torsten Seelig, MichaelV. Kurgansky, Abouzar Ghasemi V., Ion Dan Borcia, Andreas Will, Eberhard Schaller, Christoph Egbers, and Uwe Harlander. Inertial wave excitation and focusing in a liquid bounded by a frustum and a cylinder. Journal of Fluid Mechanics, 751:255297, 2014.

[22] Romain Lagrange, Christophe Eloy, Franois Nadal, and Patrice Meunier. Instability of a fluid inside a precessing cylinder. Physics of Fluids, 20(8):081701, 2008.

[23] Yufeng Lin, Jerome Noir, and Andrew Jackson. Experimental study of fluid flows in a precessing cylindrical annulus. Physics of Fluids, 26(4):046604, 2014.

[24] L. R. M. Maas, D. Benielli, J. Sommeria, and F.-P. A. Lam. Observation of an internal wave attractor in a confined, stably stratified fluid. Nature, 388:557-561, August 1997.

[25] L. R. M. Maas and F.-P. A. Lam. Geometric focusing of internal waves. Journal of Fluid Mechanics, 300:1-41, 1995.

[26] Leo R. M. Maas. Wave focusing and ensuing mean flow due to symmetry breaking in rotating fluids. Journal of Fluid Mechanics, 437:1328, 2001.

[27] Astrid Manders and Leo Maas. Observations of inertial waves in a rectangular basin with one sloping boundary. Journal of Fluid Mechanics, 493:59-88, 2003.

[28] Astrid M M Manders and Leo R M Maas. On the three-dimensional structure of the inertial wave field in a rectangular basin with one sloping boundary. Fluid Dynamics Research, 35(1):1, 2004.

[29] P. Maurer, S. J. Ghaemsaidi, S. Joubaud, T. Peacock, and P. Odier. An axisymmetric inertia-gravity wave generator. Experiments in Fluids, 58(10):143, Sep 2017.

[30] M. Mercier, N. Garnier, and T. Dauxois. Analyzing emission, reflection and diffraction of internal waves using the Hilbert transform. In APS Division of Fluid Dynamics Meeting Abstracts, page G2, November 2008.

[31] M. J. Mercier, N. B. Garnier, and T. Dauxois. Reflection and diffraction of internal waves analyzed with the Hilbert transform. Physics of Fluids, 20(8):086601, August 2008.

[32] Gordon I Ogilvie. Wave attractors and the asymptotic dissipation rate of tidal disturbances. Journal of Fluid Mechanics, 543:19-44, 2005.

[33] M. Rieutord, B. Georgeot, and L. Valdettaro. Inertial waves in a rotating spherical shell: attractors and asymptotic spectrum. Journal of Fluid Mechanics, 435:103144, 2001.

[34] M Rieutord, Bertrand Georgeot, and L Valdettaro. Wave attractors in rotating fluids: a paradigm for ill-posed cauchy problems. Physical Review Letters, 85(20):4277, 2000.

[35] M Rieutord and L Valdettaro. Viscous dissipation by tidally forced inertial modes in a rotating spherical shell. Journal of Fluid Mechanics, 643:363-394, 2010.

[36] H. Scolan, E. Ermanyuk, and T. Dauxois. Nonlinear Fate of Internal Wave Attractors. Physical Review Letters, 110(23):234501, June 2013.

[37] A. Tilgner. 8.07 - rotational dynamics of the core. In Gerald Schubert, editor, Treatise on Geophysics, pages 207 - 243. Elsevier, Amsterdam, 2007.

[38] A. Tilgner and F. H. Busse. Fluid flows in precessing spherical shells. Journal of Fluid Mechanics, 426:387396, 2001. 